\title{
Associations between cognition and serotonin in patients with major depressive disorder
}

Ämma Tangen, Jonas Svensson, Cecilia Svanborg, Christer Halldin, Jacqueline Borg \& Johan Lundberg

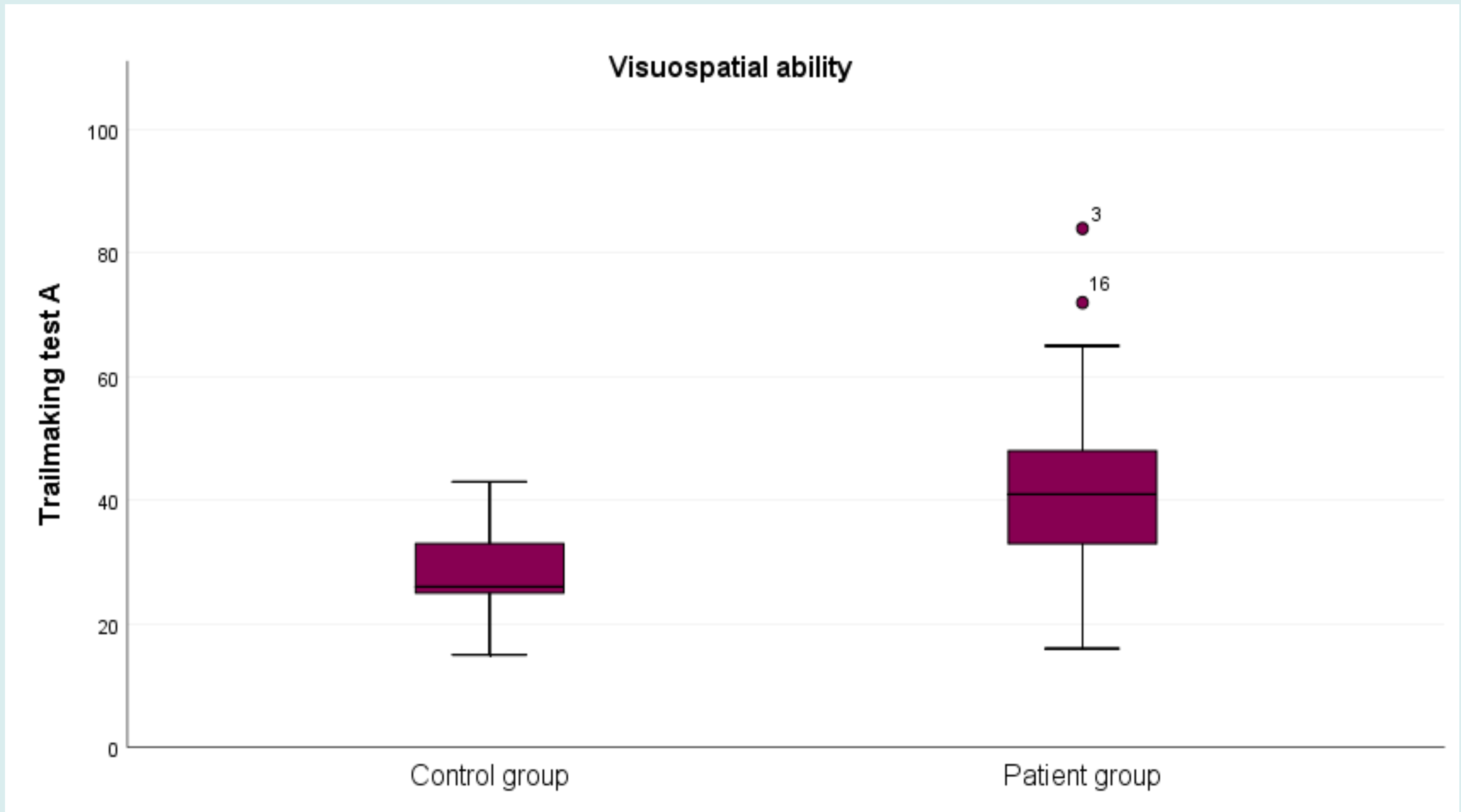

\section{Introduction}

Major depressive disorder (MDD) is a leading cause of disability worldwide ${ }^{1}$ with symptoms of low mood, loss of interest, feelings of worthlessness and cognitive impairments. Depressed patients report cognitive deficits, and several of these remain even after reduction of depressive symptoms, mainly memory, attention and executive dysfunction ${ }^{2,3}$.

The neurotransmitter of serotonin is well investigated in MDD. Animal studies and human in vivo studies report that the serotonin transporter (5-HTT) and the serotonin receptor $5-\mathrm{HT}_{1 \mathrm{~B}}$ play an important role in the disease ${ }^{4-6}$, but also specific cognitive abilities ${ }^{7}$. However, the exact roles for $5-\mathrm{HTT}$ and $5-\mathrm{HT}_{1 \mathrm{~B}}$ are still largely unknown.

\section{Method}

Seventeen patients (25-78 years; 13 females) with diagnosed MDD were examined with a cognitive battery of tests as well as positron emission tomography (PET) and the radioligands $\left[{ }^{11} \mathrm{C}\right]$ MADAM for $5-\mathrm{HTT}$ and $\left[{ }^{11} \mathrm{C}\right] \mathrm{AZ} 10419369$ for $5-\mathrm{HT}_{1 \mathrm{~B}}$ respectively, before and after Internet-based cognitive behavioral therapy, and in comparison, to an IQ, -gender,- and age matched control group.

The cognitive test battery consisted of tests that measure cognitive abilities that are affected in MDD, such as memory, visuospatial ability and executive function.

\section{Conclusion}

- The patient group was slower than the control group in tests measuring visuospatial ability.

- Improved performance in the patient group from baseline to follow-up regarding visuo-constructive ability, verbal fluency, attention and visuospatial ability.

- Analyses of putative associations between improvement in cognitive function and change in serotonin markers remain to be performed.

\section{Previous results}

A previous pilot study ${ }^{7}$ showed improvements in the patient group regarding verbal fluency $(t=-3.14$; $p=0.02$ ) from baseline to follow-up. Significant correlations were found between visuospatial ability and $5-\mathrm{HT}_{1 \mathrm{~B}}$ binding potential in several brain regions.

Furthermore, changes in cognition correlated with changes in 5- $\mathrm{HT}_{1 \mathrm{~B}}$ availability between verbal fluency and ventral striatum $(r=0.79 ; p=0.033)$, and amygdala $(r=0.76 ; p=0.049)$, as well as between visuospatial ability and the dorsal brainstem $(r=0.85 ; p=0.032)$ and hippocampus $(r=0.89 ; p=0.017)$.

\section{Preliminary results}

Statistical analyses showed a significant difference between the groups in terms of visuospatial ability $(t=3.2 ; p=0.005)$ at baseline.

Improvements in the patient group from baseline to follow-up were found in several cognitive tests, including visuo-constructive ability ( $\mathrm{t}=-4.9 ; p=0.000)$, verbal fluency $(t=-3.4 ; p=0.003)$, attention $(t=-4.7$; $p=0.000)$ and visuospatial ability $(t=3.5 ; p=0.003)$.

Statistical analyses for correlations between cognitive data and binding potential in brain regions remain to be performed.

$\begin{array}{ll}\text { References } & \\ \text { 1. WHO, } 2017 & \text { 5. Kambeitz \& Howes, } 2015 \\ \text { 2. Trivedi et al., } 2014 & \text { 6. Tiger et al., } 2014 \\ \text { 3. Bora et al., } 2013 & \text { 7. Tangen et al., } 2017 \\ \text { 4. Rock et al., } 2013 & \end{array}$

